Applied Mathematical Sciences, Vol. 10, 2016, no. 34, 1663 - 1681

HIKARI Ltd, www.m-hikari.com

http://dx.doi.org/10.12988/ams.2016.63109

\title{
Analysis of the Railway Network Operations Safety, with of Different Obstacles along the Route, by the Study of Buffon-Laplace Type Problems: the Case of Infrastructure with Irregular Hexagonal Lattices
}

\author{
Maurizio Gentile \\ CEO Rete Ferroviaria Italiana, Italy \\ Dario Lo Bosco \\ Dipartimento Meccanica e Materiali \\ Universita degli Studi Mediterranea di Reggio Calabria \\ Reggio Calabria University, Italy \\ Maria Pettineo \\ Universita degli Studi di Palermo \\ via Archirafi, 3490123 Palermo (PA), Italy \\ Copyright (c) 2016 Maurizio Gentile et al. This article is distributed under the Creative \\ Commons Attribution License, which permits unrestricted use, distribution, and reproduc- \\ tion in any medium, provided the original work is properly cited.
}

\begin{abstract}
In this paper we use an approach based on a Buffon-Laplace type problem for an irregular hexagonal lattice and obstacles to study some problems about analysis of the railway network operations safety in the presence of different obstacles on the route.
\end{abstract}

Mathematics Subject Classification: 60D05, 52A22

Keywords: Geometric Probability, stochastic geometry, random sets, random convex sets and integral geometry 


\section{Introduction}

In case of a railway transport system, referring to suitable, variable characteristics, such as the infrastructure utilization demand and the offered resistance capacity, it is possible to evaluate the related conditions of the system reliability, so that the relevant safety and operations quality standards are optimized.

In particular, the global safety of the system is structurally linked to the characteristics of reliability and vulnerability of the single line sections that constitute the network (station nodes included) where the various passenger and freight trains run; so, if we analyze the generic $j$ - $n$th route on the network (taking into account the general effects produced by each i- $n$th component that constitutes it, with $\mathrm{i}=1, \ldots, \mathrm{n})$ among the $m$ routes that form the $K$ network altogether and that are characterizing the examined optimization problem, the two above mentioned resulting indicators assume, respectively, the form of:

$$
D_{g}=\sum_{j=1}^{m} D_{g j}, \quad R_{g}=\sum_{j=1}^{m} R_{g j} .
$$

From the vectorial point of view, the (1), built the related column vectors $(n$ order $)$

$$
\vec{D}_{g j} \equiv\left\|\begin{array}{c}
D_{g 1} \\
\vdots \\
D_{g i} \\
\vdots \\
D_{g n}
\end{array}\right\|, \quad \vec{R}_{g j} \equiv\|\| \begin{gathered}
R_{g 1} \\
\vdots \\
R_{g i} \\
\vdots \\
R_{g n}
\end{gathered} \|
$$

and considering the whole of $\mathrm{m}$ component vectors, we have

$$
\vec{D}_{g}=\left\{\vec{D}_{g j}\right\}_{j=1}^{m} e \vec{R}_{g}=\left\{\vec{R}_{g j}\right\}_{j=1}^{m}
$$

On the basis of the obtained variables values (vectors moduli), it is easier to check the compliance of two particular conditions characterizing the studied system: $\boldsymbol{M}=\boldsymbol{R}-\boldsymbol{D}>\boldsymbol{O}$ (safety margin); $\mathbf{F}=\boldsymbol{R} / \boldsymbol{D}>\boldsymbol{1}$ (safety factor).

Known the probability functions of the aforesaid random variables, the probability that a vulnerability limit status is reached, is expressed by the integral sum of probabilities that the safety factor $\mathbf{F}$ is included in the interval $[0,1]$,

$$
\mathbf{P}_{\mathbf{r}}=\int_{\mathbf{0}}^{\mathbf{1}} f_{\phi}(\Phi) \mathrm{d} \Phi
$$

where $f_{\Phi}$ is the density function of the variable $F$ probability, while the corresponding reliability is measured by the expression: $P a=1-\operatorname{Pr}$. 
Possible anomalies along the line, such as the presence of obstacles of different size and kind that may occupy the railway track, represent further criticalities for safety, whose effects can be, however, previously and suitably analyzed, also in order to outline proper alternative routes to assure, in case of emergency, the transport service until the foreseen final destination, for each Origin Destination relation (O-D matrix) interesting the customers.

This is possible, for example, if we refer to a particular mathematical characterization of the problem, using a methodological approach based on stochastic geometry and geometric probabilities elements and by building a special graph representing the network (fig. 1).

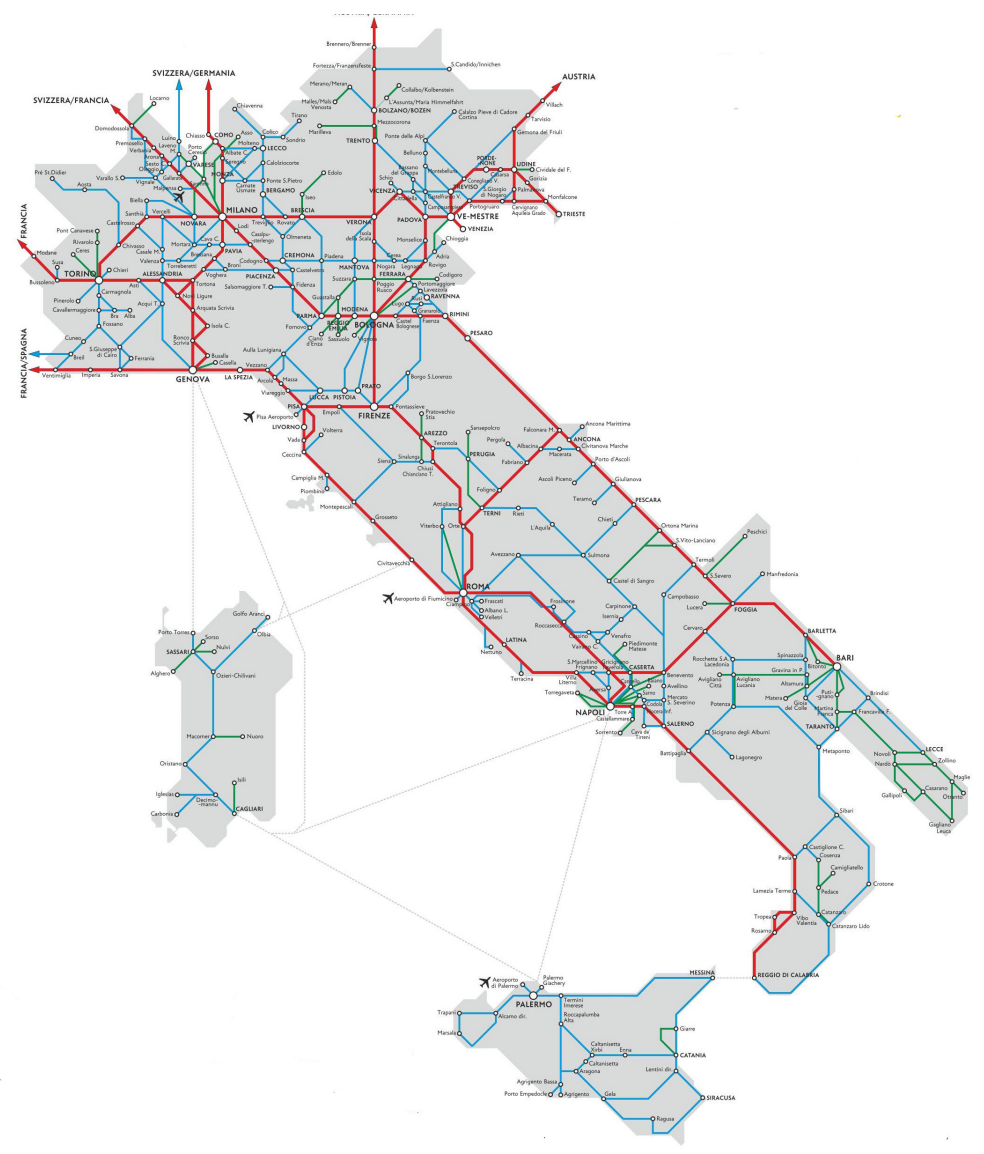

Fig. 1 - Example of a typical railway network graph

This will be formed by a union set of geometrical figures forming particular $\Re$ lattices made up - in the most general case dealt with by this paper - also by irregular geometric figures (we are now going to consider, as an example, irregular hexagons) - in the referred $S m$ geometrical space. With particular test bodies (mathematic models), representing the trains, it is so possible to study the relative motion in $\Re$ and the possible interferences on them generated 
by obstacles (of different form and size) along each section $h-k$ of the considered network.

In the railway field, these test bodies, in the analysis we are going to carry out, can be assumed as segments of suitable length $l$ (in order to schematize a train with a high number of carriages, such as for a freight train, etc.), or formed by rectangles with $l 1$ e $l 2$ sides (like in the case of a high speed train, a regional train, etc.).

In order to find the mathematical solution of the problem, in the following approach we will assume, however, that each side of the lattice give the same resilience of the advancement of the test body. More, $\Re$ will take the form of an irregular hexagon to generalize the problem, that as has been already studied with by the Authors in previous papers concerning cases with regular geometric figures only), while as to the obstacles on the railway track interfering with the train running, they will take a different generic form, both triangular and circular (e.g. representing the section of a broken tree fallen on the track, etc.); finally, it will be used a constant length segment $l$ as test body, in order to explain the studied problem more easily.

\section{The mathematical characterization of the generalized problem through lattices with irregular obstacles placed on the railway track.}

Let $\Re(a, \alpha, m)$ be the considered irregular lattice, with the fundamental cell $\mathcal{C}_{0}$ represented in figure 1 where $\alpha \in\left[\frac{\pi}{4}, \frac{\pi}{2}\right.$ [ and where the obstacles are isosceles triangles with $m / 2, m / 2$ sides and circular sectors with $m / 2$ radius, with $0 \leq$ $m<\min (a, \operatorname{atg} \alpha)$.

It results from fig. 2: 


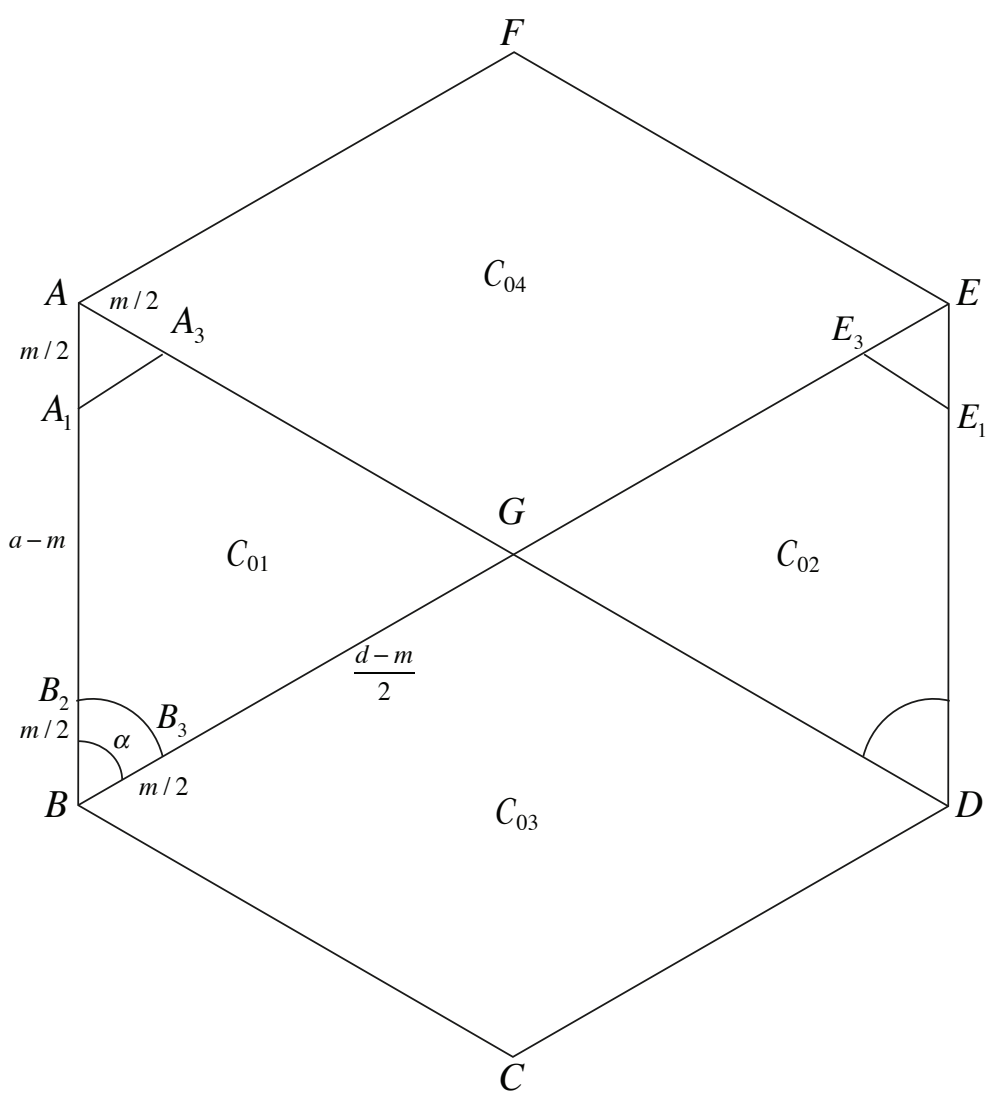

Fig. 2

$$
\begin{gathered}
d=\frac{a}{\cos \alpha},|B C|=|C D|=|A F|=|E F|=|A G|= \\
|B G|=|D G|=|E G|=\frac{d}{2}, \\
\widehat{A G B}=\widehat{E G D}=\pi-2 \alpha, \widehat{A G E}=\widehat{B G D}=2 \alpha \\
\widehat{B_{1} B B_{3}}=\widehat{A_{2} A A_{3}}=\widehat{E_{2} E E_{3}}=\widehat{D_{1} D D_{3}}=\pi-2 \alpha, \widehat{A_{1} A A_{3}}= \\
\widehat{B_{2} B B_{3}}=\widehat{D_{2} D D_{3}}=\widehat{E_{1} E E_{3}}=\alpha, \\
\left|A_{1} A_{3}\right|=\left|E_{1} E_{3}\right|=m \sin \frac{\alpha}{2},\left|A_{2} A_{3}\right|=\left|B_{1} B_{3}\right|=\left|D_{1} D_{3}\right|=\left|E_{2} E_{3}\right|=m \cos \alpha \\
\text { areaA } A_{1} A_{3}=\text { areaE } E_{1} E_{3}=\frac{m^{2}}{8} \sin \alpha, \text { areaB } B_{2} B_{3}=\text { areaD } D_{2} D_{3}=\frac{m^{2} \alpha}{8}
\end{gathered}
$$


area $A A_{2} A_{3}=$ area $B B_{1} B_{3}=$ area $D D_{1} D_{3}=$ area $E E_{2} E_{3}=\frac{m^{2}}{8} \sin 2 \alpha$.

With these values we obtain

$$
\begin{aligned}
& \text { area } \mathcal{C}_{01}=\text { areaC } \mathcal{C}_{02}=\frac{a^{2}}{4} \operatorname{tg} \alpha-\frac{m^{2}}{8}(\sin \alpha+\alpha), \\
& \text { area } \mathcal{C}_{03}=\text { area } \mathcal{C}_{04}=\frac{a^{2}}{2} \operatorname{tg} \alpha-\frac{m^{2}}{4}(\sin 2 \alpha), \\
& \text { area } \mathcal{C}_{0}=\frac{3 a^{2}}{2} \operatorname{tg} \alpha-\frac{m^{2}}{8}(2 \sin 2 \alpha+\sin \alpha+\alpha) .
\end{aligned}
$$

We now consider a segment $s$ with random position and constant length

$l<\min (a-m, a t g \alpha-m)$ and we want to determine the probability that this segment intersects one side of $\mathcal{R}$ reticule; this probability is equal to $P_{\text {int }}$ probability that segment $s$ intersects one side of lattice.

The position of segment $s$ is given by itscenter and by the angle $\varphi$ that it forms with $B C$ side of the cell $\mathcal{C}_{0}$.

We calculate the probability $P_{\text {int }}$ considering the limit positions of segment $s$, for a set value of $\varphi$, in cell $\mathcal{C}_{0 i},(i=1,2,3,4)$. fig. 3

Indicating with $\widehat{\mathcal{C}_{0 i}}(\varphi)$ the polygon determined by these positions we have 


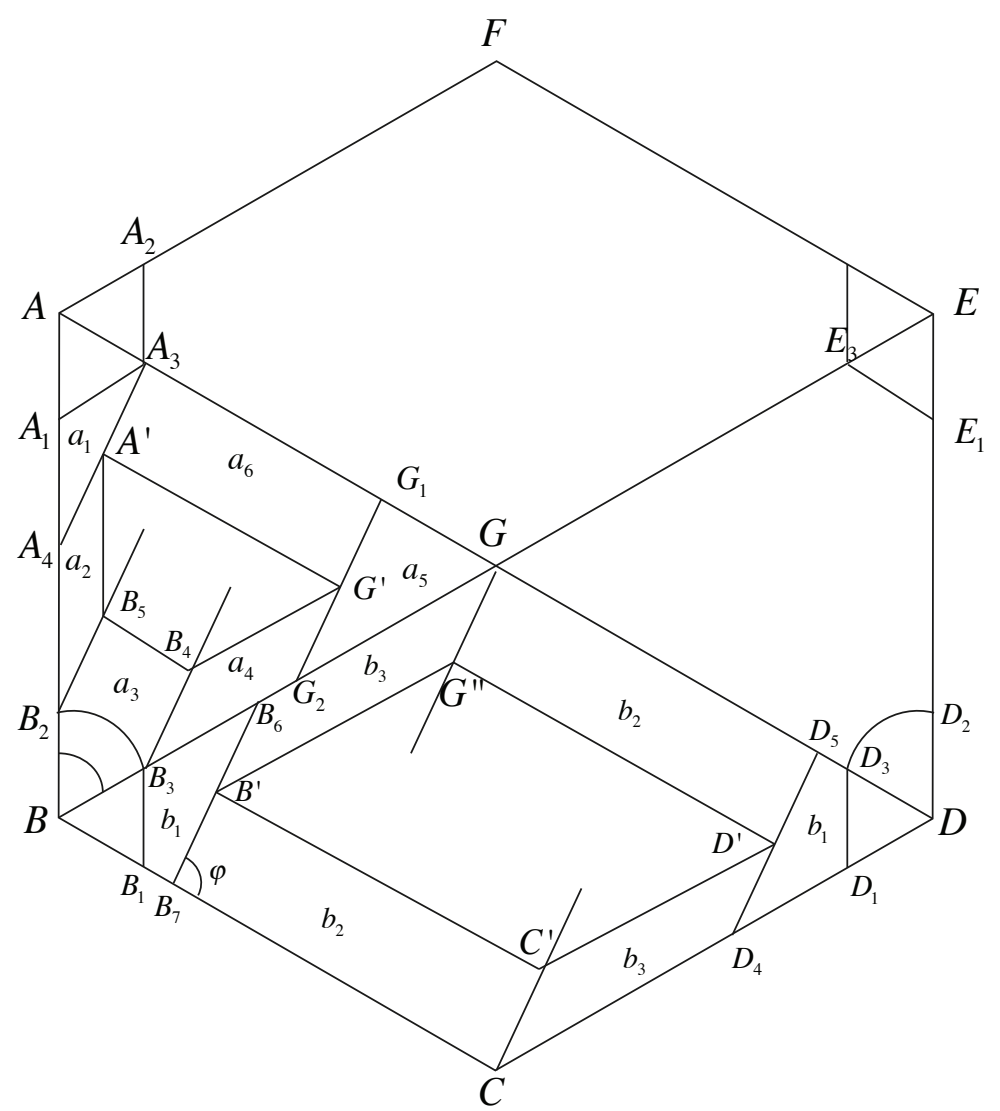

Fig. 3

Taking into account this figure, we have

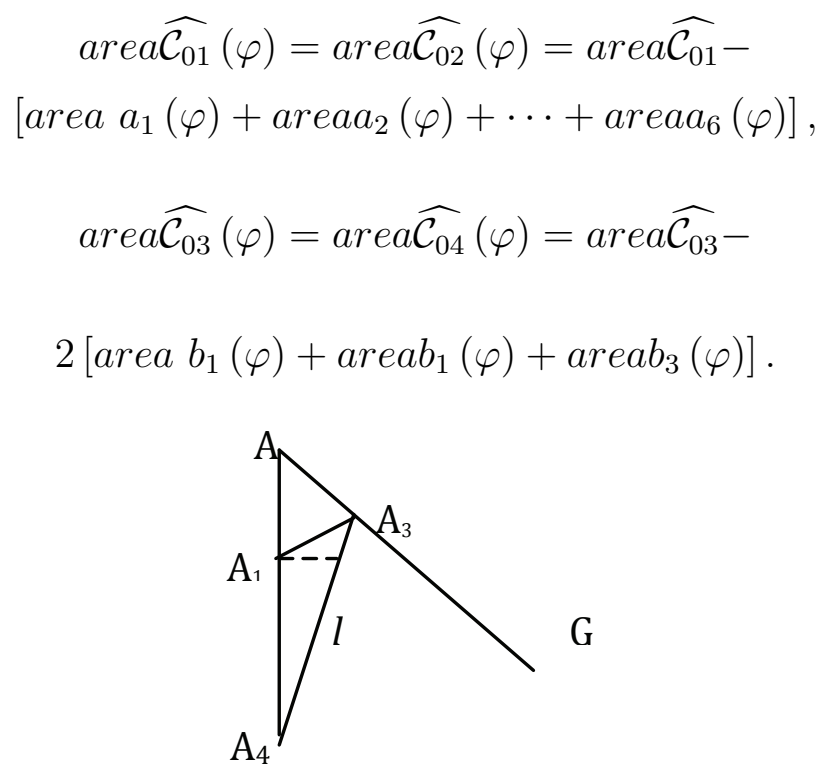

Fig. 4 
By fig. 4 it results

$$
\widehat{A_{4} A_{3} G}=\varphi, \widehat{A_{4} A_{3} A}=\pi-\varphi, \widehat{A A_{1} A_{3}}=\widehat{A A_{3} A_{1}}=\frac{\pi}{2}-\frac{\alpha}{2}, \widehat{A_{4} A_{1} A_{3}} \frac{\pi}{2}+\frac{\alpha}{2},
$$

1.

$$
\widehat{A_{4} A_{3} A_{1}}=\frac{\pi}{2}-\varphi+\frac{\alpha}{2}, \quad \widehat{A_{1} A_{4} A_{3}}=\varphi-\alpha
$$

From triangle $A_{1} A_{3} A_{4}$ and with (4) we have

$$
\frac{\left|A_{1} A_{4}\right|}{\cos \left(\varphi-\frac{\alpha}{2}\right)}=\frac{l}{\cos \frac{\alpha}{2}}=\frac{m \sin \frac{\alpha}{2}}{\sin (\varphi-\alpha)}
$$

SO

$$
\left|A_{1} A_{4}\right|=\frac{l \cos \left(\varphi-\frac{\alpha}{2}\right)}{\cos \frac{\alpha}{2}}
$$

and the condition

$$
m \sin \alpha=2 l \sin (\varphi-\alpha) .
$$

then, with (9), we have

$$
h_{1}=\left|A_{1} A_{4}\right| \cdot \sin {\widehat{A_{1} A_{4} A_{3}}}_{3}=\frac{l \cos \left(\varphi-\frac{\alpha}{2}\right) \sin (\varphi-\alpha)}{\cos \frac{\alpha}{2}} .
$$

therefore

$$
\text { area } a_{1}(\varphi)=\frac{l^{2} \cos \left(\varphi-\frac{\alpha}{2}\right) \sin (\varphi-\alpha)}{2 \cos \frac{\alpha}{2}} .
$$

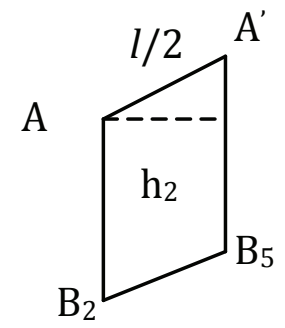

Fig. 5

From fig. 5

$$
{\widehat{A_{4} B_{2} B_{5}}}={\widehat{A_{1} A_{4} A_{3}}}_{3}=\varphi-\alpha
$$




$$
h_{2}=\frac{l}{2} \sin (\varphi-\alpha)
$$

Then

$$
\left|A_{4} B_{2}\right|=a-m-\left|A_{1} A_{4}\right|=a-m-\frac{l \cos \left(\varphi-\frac{\alpha}{2}\right)}{\cos \frac{\alpha}{2}},
$$

it results

$$
\text { area } a_{2}(\varphi)=\left[a-m-\frac{l \cos \varphi-\frac{\alpha}{2}}{\cos \frac{\alpha}{2}}\right] \cdot \frac{l}{2} \sin (\varphi-\alpha) .
$$

The relations (12) and (13) give

$$
\text { area } a_{1}(\varphi)+\text { area } a_{2}(\varphi)=(a-m) \cdot \frac{l}{2} \sin (\varphi-\alpha)
$$

In order to calculate area $a_{3}(\varphi)$, we consider following figure

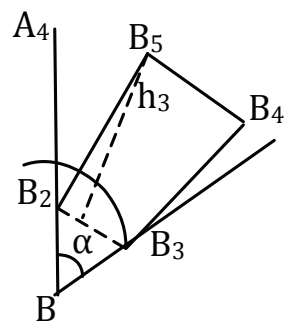

Fig. 6

We have

$$
\widehat{B_{5} B_{2} B_{3}}=\pi-\left(\widehat{A_{4} B_{2} B_{5}}+\frac{\pi}{2}-\frac{\alpha}{2}\right)=\frac{\pi}{2}-\varphi+\frac{3 \alpha}{2},
$$

SO

$$
h_{3}=\frac{l}{2} \sin \widehat{B_{3} B_{2} B_{5}}=\frac{l}{2} \cos \left(\varphi-\frac{3 \alpha}{2}\right) .
$$

Furthermore, with (5) we have

$$
\text { circular segment area } B_{2} B_{3}=\frac{m^{2} \alpha}{8}-\frac{m^{2}}{8} \sin \alpha,
$$

therefore

$$
\text { area } a_{3}(\varphi)=\frac{m l \sin \frac{\alpha}{2}}{2} \cos \left(\varphi-\frac{3 \alpha}{2}\right)-\frac{m^{2}}{8}(\alpha-\sin \alpha) .
$$




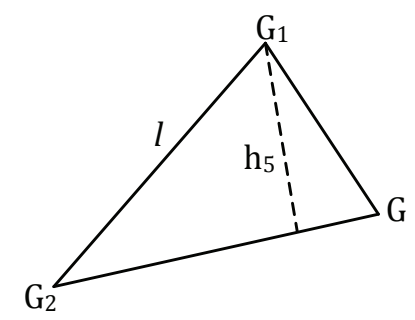

Fig. 7

Now from fig. 7 we have

$$
\begin{aligned}
& \widehat{G_{1} G G_{2}}=\pi-2 \alpha, \widehat{G_{1} G_{2} G}=\widehat{B_{4} B_{3} G}= \\
& \pi-\left(\frac{\pi}{2}-\frac{\alpha}{2}+\pi-\widehat{B_{5} B_{2} B_{3}}\right)=2 \alpha-\varphi
\end{aligned}
$$

SO

$$
h_{5}=l \sin (2 \alpha-\varphi) .
$$

But given $G_{1} G_{2} \| B_{6} B_{7}$ e $G G_{1} \| B C$, it follows

$$
\widehat{G G_{1} G_{2}}=\varphi
$$

So the triangle ${\widehat{G G_{1} G_{2}}}_{2}$ gives

$$
\frac{\left|G G_{1}\right|}{\sin (2 \alpha-\varphi)}=\frac{\left|G G_{2}\right|}{\sin \varphi}=\frac{l}{\sin 2 \alpha},
$$

SO

$$
\left|G G_{1}\right|=\frac{l \sin (2 \alpha-\varphi)}{\sin 2 \alpha},\left|G G_{2}\right|=\frac{l \sin \varphi}{\sin 2 \alpha}
$$

Therefore

$$
\text { area } a_{5}(\varphi)=\frac{l^{2} \sin \varphi \sin (2 \alpha-\varphi)}{2 \sin 2 a}
$$

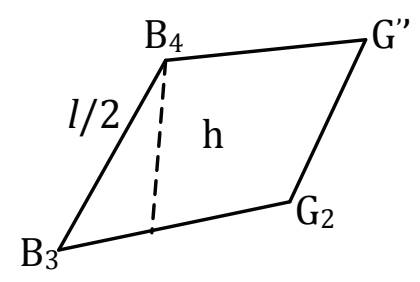

Fig. 8 
Figure 8 gives

$$
\widehat{B_{4} B_{3} G_{2}}=2 \alpha-\varphi, h_{4}=\frac{l}{2} \sin (2 \alpha-\varphi) .
$$

Furthermore, with (16), we have

$$
\left|B_{3} G_{2}\right|=\frac{d}{2}-\frac{m}{2}-\left|G G_{2}\right|=\frac{a}{2 \cos \alpha}-\frac{m}{2}-\frac{l \sin \varphi}{2 \sin \alpha} .
$$

So

$$
\text { area } a_{4}(\varphi)=\left(\frac{a}{2 \cos \alpha}-\frac{m}{2}-\frac{\operatorname{lsin} \varphi}{\sin 2 \alpha}\right) \cdot \frac{l}{2} \sin (2 \alpha-\varphi) .
$$

The relations (17) and (18) give

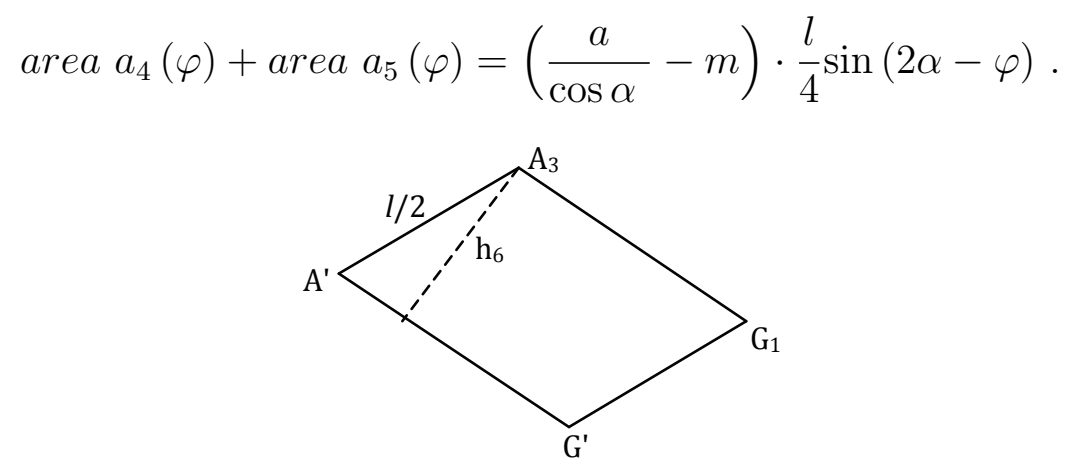

Fig. 9

By figure 9 it results that

$$
\widehat{A_{3} G_{1} G^{\prime}}=\pi-{\widehat{G G_{1} G_{2}}}^{\prime}=\varphi, h_{6}=\frac{l}{2} \sin \varphi
$$

and, with (16),

$$
\left|A_{3} G_{1}\right|=\frac{d}{2}-\frac{m}{2}-\left|G G_{1}\right|=\frac{a}{2 \cos \alpha}-\frac{m}{2}-\frac{l \sin (2 \alpha-\varphi)}{\sin 2 \alpha} .
$$

As a consequence

$$
\text { area } a_{6}(\varphi)=\left[\frac{a}{2 \cos \alpha}-\frac{m}{2}-\frac{l \sin (2 \alpha-\varphi)}{\sin 2 \alpha}\right] \cdot \frac{l}{2} \sin \varphi .
$$

Replacing in (7) the expressions (14), (15), (19) and (20) we obtain

$$
\begin{aligned}
& \text { area } \widehat{\mathcal{C}}_{01}(\varphi)=\text { area } \widehat{\mathcal{C}}_{02}(\varphi)=\text { area } \mathcal{C}_{01}- \\
& {\left[\frac{m l}{4} \sin \alpha \cos \varphi+\frac{a-m \cos ^{2} \alpha}{\cos \alpha} \cdot \frac{l}{4} \sin \varphi-\right.}
\end{aligned}
$$




$$
\left.-\frac{l^{2}}{4 \sin 2 \alpha}(\cos 2 \alpha \cos 2 \varphi+\sin 2 \alpha \sin 2 \varphi-\cos 2 \alpha \quad)-\frac{m^{2}}{8}(\alpha-\sin \alpha)\right]
$$

We now consider area $\widehat{\mathcal{C}}_{03}(\varphi)$.

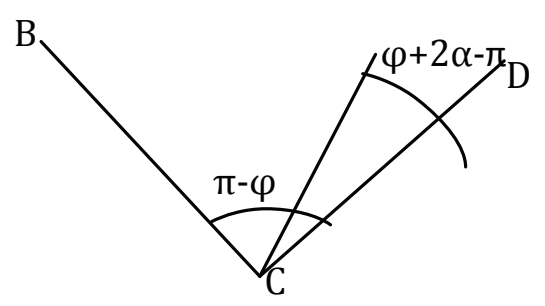

Fig. 10

From figure 10

$$
\varphi_{1}=\pi-2 \alpha, \varphi_{2}=2 \alpha
$$

therefore

$$
\varphi \in[\pi-2 \alpha, 2 \alpha]
$$

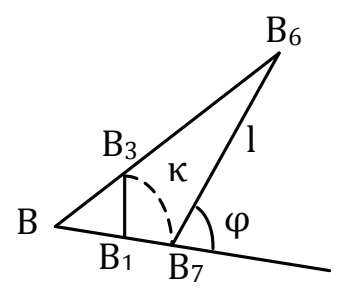

Fig. 11

Figure 11 and the relations (3) give

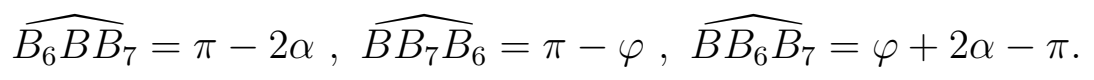

From triangle $\mathrm{BB}_{6} B_{7}$ it results

$$
\frac{\left|B B_{6}\right|}{\sin \varphi}=\frac{\left|B B_{7}\right|}{\sin (\varphi+2 \alpha-\pi)}=\frac{l}{\sin 2 \alpha}
$$

that is,

$$
\left|B B_{6}\right|=\frac{l \sin \varphi}{\sin 2 \alpha}, \quad\left|B B_{7}\right|=\frac{l \sin (\varphi+2 \alpha-\pi)}{\sin 2 \alpha} .
$$

In view of (23) we can state 


$$
\sin (\varphi+2 \alpha-\pi) \geq 0
$$

So

$$
\left|B B_{7}\right|=-\frac{l \sin (\varphi+2 \alpha)}{\sin 2 \alpha} \geq 0 \text {. }
$$

furthermore,

$$
\kappa_{1}=l \sin {\widehat{B B_{6} B_{7}}}_{7}=l \sin (\varphi+2 \alpha-\pi)=-l \sin (\varphi+2 \alpha)
$$

And taking into account (5)

$$
\text { area } b_{1}(\varphi)=-\frac{l^{2} \sin \varphi \sin (\varphi+2 \alpha)}{2 \sin 2 \alpha}-\frac{m^{2}}{8} \sin 2 \alpha .
$$

From figure 12

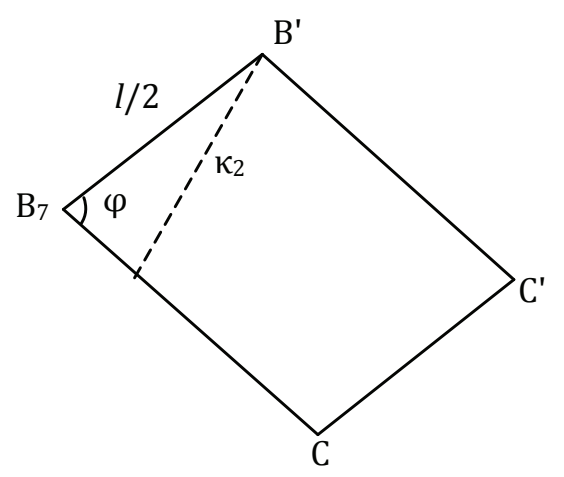

Fig. 12

we have that

$$
\kappa_{2}=\frac{l}{2} \sin \varphi
$$

and in view of (25)

$$
\left|B_{7} C\right|=\frac{d}{2}-\left|B B_{7}\right|=\frac{a}{2 \cos \alpha}+\frac{l \sin (\varphi+2 \alpha)}{\sin 2 \alpha} .
$$

Therefore,

$$
\text { area } b_{2}(\varphi)=\frac{\left|B_{7} C\right| \cdot \kappa_{2}}{2}
$$

that is,

$$
\text { area } b_{2}(\varphi)=\left[\frac{a}{2 \cos \alpha}+\frac{l \sin (\varphi+2 \alpha)}{\sin 2 \alpha}\right] \cdot \frac{l}{2} \sin \varphi
$$


The formulae (26) and (27) give

$$
\text { area } b_{1}(\varphi)+\text { area } b_{2}(\varphi)=\frac{a l \sin \varphi}{4 \cos \alpha}-\frac{m^{2}}{8} \sin 2 \alpha \text {. }
$$

Finally figure 13

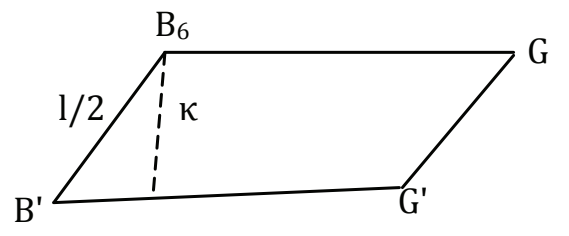

Fig. 13

gives

$$
\widehat{B_{6} B^{\prime} G^{\prime}}=\widehat{B_{4} B_{3} G}=2 \alpha-\varphi, \kappa_{3}=\frac{l}{2} \sin (2 \alpha-\varphi) .
$$

Taking into consideration (24) we have

so

$$
\left|B_{6} G\right|=\frac{d}{2}-\left|B B_{6}\right|=\frac{a}{2 \cos \alpha}-\frac{l \sin \varphi}{\sin 2 \alpha}
$$

$$
\text { area } b_{3}(\varphi)=\left(\frac{a}{2 \cos \alpha}-\frac{l \sin \varphi}{\sin 2 \alpha}\right) \cdot \frac{l}{2} \sin (2 \alpha-\varphi) \text {. }
$$

Replacing in (8) the expressions (28) and (29) we obtain

$$
\begin{gathered}
\text { area } \widehat{\mathcal{C}}_{03}(\varphi)=\text { area } \mathcal{C}_{04}(\varphi)=\text { area } \mathcal{C}_{03}- \\
{\left[\operatorname{alsin} \alpha(\cos \varphi+\operatorname{tg} \alpha \sin \varphi)-\frac{l^{2}}{2}\right.} \\
\left.(\sin 2 \varphi++\operatorname{ctg} 2 \alpha \cos 2 \varphi-\operatorname{ctg} 2 \alpha)-\frac{m^{2}}{4} \sin 2 \alpha\right] .
\end{gathered}
$$

Indicating as $\mathcal{M}_{i}$ the whole of s segments that have the medium point in $\mathcal{C}_{0 i},(i=1,2,3,4)$ and as $\mathcal{N}_{i}$ the whole of s segments fully contained in $\mathcal{C}_{0 i}$, we have [15]:

$$
\mathrm{P}_{\text {int }}=1-\frac{\mu\left(\mathcal{N}_{1}\right)+\mu\left(\mathcal{N}_{2}\right)+\mu\left(\mathcal{N}_{3}\right)+\mu\left(\mathcal{N}_{4}\right)}{\mu\left(\mathcal{M}_{1}\right)+\mu\left(\mathcal{M}_{2}\right)+\mu\left(\mathcal{M}_{3}\right)+\mu\left(\mathcal{M}_{4}\right)},
$$

where $\mu$ is the Lebesgue measure in the Euclidean plan

The measures $\mu\left(\mathcal{M}_{i}\right)$ e $\mu\left(\mathcal{N}_{i}\right)$ are calculated by using the Poincaré [14] kinematic measure: 


$$
d k=d x \wedge d y \wedge d \varphi
$$

where $\mathrm{x}, \mathrm{y}$ are the coordinates of point 0 barycenter and $\varphi$ the already defined angle.

In order to calculate the measures $\mu\left(\mathcal{M}_{1}\right), \mu\left(\mathcal{M}_{2}\right), \mu\left(\mathcal{N}_{1}\right)$ e $\mu\left(\mathcal{N}_{2}\right)$, we consider figure

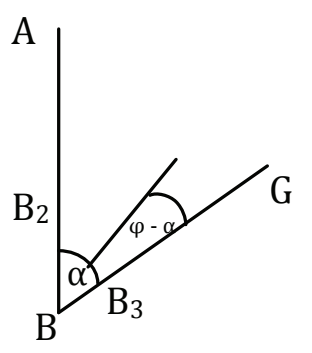

Fig. 14

Hence

$$
\varphi_{1}-\alpha=0, \quad \varphi_{2}-\alpha=\alpha,
$$

therefore, for cells $\mathcal{C}_{01}$ e $\mathcal{C}_{02}$, we have

$$
\varphi_{1}=\alpha, \quad \varphi_{2}=2 \alpha,
$$

that is

$$
\varphi \in[\alpha, 2 \alpha] .
$$

With these values we can state

$$
\begin{aligned}
\mu\left(\mathcal{M}_{1}\right) & =\mu\left(\mathcal{M}_{2}\right)=\int_{\alpha}^{2 \alpha} d \varphi \iint_{\left\{(x, y) \in \mathcal{C}_{01}\right\}} d x d y= \\
& =\int_{\alpha}^{2 \alpha}\left(\text { area }_{01}\right) d \varphi=\alpha \operatorname{areac}_{01}
\end{aligned}
$$

and, taking into account the formula (21), 


$$
\begin{aligned}
\mu\left(\mathcal{N}_{1}\right)= & \mu\left(\mathcal{N}_{2}\right)=\int_{\alpha}^{2 \alpha} d \varphi \iint_{\left\{(x, y) \in \widehat{\mathcal{C}}_{01}(\varphi)\right\}} d x d y= \\
= & \int_{\alpha}^{2 \alpha}\left[\operatorname{area} \widehat{\mathcal{C}}_{01}(\varphi)\right] d \varphi=\alpha \operatorname{area\mathcal {C}_{01}-} \\
& \left\{\frac{l}{4}\left[\frac{\cos \alpha-\cos 2 \alpha}{\cos \alpha} \cdot \alpha-m(1+\cos \alpha)\right]-\right. \\
& \left.--\frac{l^{2}}{8}(1-2 \alpha \operatorname{ctg} 2 \alpha)-\frac{m^{2}}{8} \alpha(\alpha-\sin \alpha)\right\}
\end{aligned}
$$

Then, taking into account the formulae (22) and (30), we have

$$
\begin{aligned}
& \mu\left(\mathcal{M}_{3}\right)=\mu\left(\mathcal{M}_{4}\right)=\int_{\pi-2 \alpha}^{2 \alpha} d \varphi \iint_{\left\{(x, y) \in \mathcal{C}_{03}\right\}} d x d y= \\
& =\int_{\pi-2 \alpha}^{2 \alpha}\left(\operatorname{area}_{03}\right) d \varphi=(4 \alpha-\pi) \operatorname{areaC}_{03}, \\
& \mu\left(\mathcal{N}_{3}\right)=\mu\left(\mathcal{N}_{4}\right)=\int_{\pi-2 \alpha}^{2 \alpha} \iint_{\left\{(x, y) \in \widehat{\mathcal{C}}_{03}(\varphi)\right\}} d x d y= \\
& =\int_{\pi-2 \alpha}^{2 \alpha}\left[\operatorname{area} \widehat{\mathcal{C}}_{03}(\varphi)\right] d \varphi=(4 \alpha-\pi) \operatorname{areaC}_{03}+ \\
& +2 a l \sin \alpha \operatorname{tg} \alpha \cos 2 \alpha+\frac{l^{2}}{4}\left[4 \cos ^{2} 2 \alpha-(4 \alpha-\pi) \operatorname{ctg} 2 \alpha\right] \\
& +\frac{m^{2}}{4}(4 \alpha-\pi) \sin 2 \alpha
\end{aligned}
$$

The relations (31), (32), (33), (34) and (35) give

$$
\begin{aligned}
\mathrm{P}_{\text {int }}= & \frac{4}{a^{2}(9 \alpha-2 \pi) \operatorname{tg} \alpha-\frac{m^{2}}{2}[\alpha(\alpha+\sin \alpha)+2(4 \alpha-\pi) \sin 2 \alpha]} . \\
& \cdot\left\{\frac{l}{4}\left[\frac{a}{\cos \alpha}\left(\cos \alpha-\cos 2 \alpha-8 \sin ^{2} \alpha \cos 2 \alpha\right)-m(1+\cos \alpha)\right]\right. \\
& +\frac{l^{2}}{8}\left[8 \cos ^{2} 2 \alpha+2 \alpha \operatorname{ctg} 2 \alpha-1+(8 \alpha-2 \pi) \operatorname{ctg} 2 \alpha\right]- \\
& \left.\quad-\frac{m^{2}}{8}[\alpha(\alpha-\sin \alpha)+(8 \alpha-2 \pi) \sin 2 \alpha]\right\}
\end{aligned}
$$


In particular, for $\mathrm{m}=0$ e $\alpha=\frac{\pi}{4}$, we obtain the researched probability

$$
\underline{P}=\frac{4 \sqrt{2}}{\pi} \cdot \frac{l}{a}-\frac{2}{\pi} \cdot\left(\frac{l}{a}\right)^{2}
$$

\section{Conclusions}

The goal of this paper was the construction of suitable mathematic relations aimed at carrying out an adequate analysis of the possible interference of trains running at a given speed $V$ (supposed to be constant) on a given element of the network (belt line, node, etc.), with the possible presence of obstacles of different forms.

The achieved results allow the extension of the study on the investigated geometrical probabilities to the more general case of complex lattice with obstacles with irregular geometric form along the route of running trains (as in fig. 1 graph), really representative of the railway transport network that, in practice, will have to be examined in order to evaluate the possible interferences, so that the various profiles of railway safety can be fully optimized.

In this case, the calculation will have to consider also the rotation of angle $\mathrm{j}$ between the axis $\mathrm{Ox}$ and the line supporting the test body $\mathrm{s}$, as resulting from the expression (5).

Finally, the variable $l$ will have to be replaced with a suitable value, to be found in function of the particular case study and depending on the specific type and characteristics of the train (e.g., freight or passenger trains.

\section{References}

[1] D. Barilla, G. Caristi, A. Puglisi, M. Stoka, Laplace type problems for a triangular lattice and different body test, Applied Mathematical Sciences, 8 (2014), no. 103, 5123 - 5131.

http://dx.doi.org/10.12988/ams.2014.46423

[2] D. Barilla, G. Caristi, A. Puglisi, A Buffon - Laplace type problems for an irregular lattice and with maximum probability, Applied Mathematical Sciences, 8 (2014), no. 165, 8287 - 8293.

http://dx.doi.org/10.12988/ams.2014.411916

[3] D. Barilla, G. Caristi, E. Saitta, A Laplace type problems for a lattice with cell composed by three quadrilaterals and with maximum probability, Applied Mathematical Sciences, 8 (2014), no. 165, 8279 - 8286.

http://dx.doi.org/10.12988/ams.2014.411915 
[4] D. Barilla, G. Caristi, A. Puglisi, A Laplace type problem for a regular lattice with six obstacles, Recent Advances in Applied \\& Biomedical Informatics and Computational Engineering in System Applications, Proceedings of the 11th WSEAS International Conference on Applied Informatics and Communications, (AIC Õ11) Florence (Italy), 30-35, 2011.

[5] D. Barilla, G. Caristi, A. Puglisi, M. Stoka, A Laplace type problem for two hexagonal lattices of Delone with obstacles, Applied Mathematical Sciences, 7 (2013), no. 92, 4571 - 4581.

http://dx.doi.org/10.12988/ams.2013.35242

[6] G. Caristi, E. L. Sorte, M. Stoka, Laplace problems for regular lattices with three different types of obstacles, Applied Mathematical Sciences, 5 (2011), no. 53-56, 2765-2773.

[7] G. Caristi, A. Puglisi, M. Stoka, A Laplace type problem for a regular lattices with octagonal cell, Far East Journal Mathematical Sciences, 48 (2011), no. 1, 103-118.

[8] G. Caristi, E. Saitta, M. Stoka, Laplace type problems for some regular lattices and body test disc, International Journal of Mathematical Analysis, 9 (2015), no. 39, 1901-1917.

http://dx.doi.org/10.12988/ijma.2015.56167

[9] G. Caristi, M. Stoka, A Laplace type problem for a regular lattices with irregular hexagonal cell, Far East Journal Mathematical Sciences, 50 (2011), no. 1, 23-36.

[10] G. Caristi, M. Stoka, A Laplace type problem for a Dirichlet-Voronoi lattice different obstacles, Applied Mathematical Sciences, 5 (2011), no. 30, 1493-1523.

[11] G. Caristi, M. Stoka, A Laplace type problem for regular lattice with convex-concave cell with obstacles rhombus and circular sections, International Journal of Mathematical Analysis, 8 (2014), 1689 - 1695.

http://dx.doi.org/10.12988/ijma.2014.46165

[12] G. Caristi, M. Stoka, A Laplace type problem for a lattice with axial symmetric and different obstacles (I), Far East Journal of Mathematical Sciences, 58 (2011), no. 1, 99-118.

[13] G. Caristi and M. Stoka, A Laplace type problem for a regular lattice with obstacles (I), Atti Acc. Sci. Torino, to appear.

[14] H. Poincaré, Calcul Des Probabilitiés, ed. 2, Carré, Paris, 1912. 
[15] M. Stoka, Probabilités géométriques de type Buffon dans le plan euclidean, Atti Acc. Scienze Torino, 110 (1975-1976), 53-59.

[16] A. Duma, M. Stoka, Geometric probabilities for non convex lattices, Pub. Ist. Stat. Univ. Paris, XXXXIV, fasc. 1, 2000, p. 3546.

[17] D. Lo Bosco, F. G. Praticò, A stochastic method for shape property analysis of mineral aggregates used in road constructions: the proposal for a model and the study of hard grains, Seminarberichteaus dem Fachbereich Mathematik, band 70, 2001.

[18] A. Duma, M. Stoka, Problems of 'Buffon type' for polygonal strips, Elemente Der Mathematik, 58 (2003), 1-5.

http://dx.doi.org/10.1007/s000170300000

[19] AA. VV.,RFI [Argomenti] nn.1,4, 2003 - 2204. AA. VV.,FS [Argomenti], dicembre 2009.

[20] D. Lo Bosco, Un modello matematico per l'analisi della sicurezza e qualità globale d'esercizio per la rete ed i nodi ferroviari : lo studio delle interazioni veicolo-binario, General Meeting EFRTC - Florence, 3 December 2010 .

[21] D. Lo Bosco, A. Triolo, Un modello matematico per lo studio del comfort percepito nel trasporto ferroviario, atti dei seminari del Master di II livello in Infrastrutture e Sistemi Ferroviari, Università degli Studi di Roma "La Sapienza", 2013.

\section{Received: April 1, 2016; Published: May 12, 2016}

